\title{
Effects of transcranial direct current stimulation upon attention and visuoperceptual function in Lewy body dementia: a preliminary study
}

\author{
Greg J. Elder, ${ }^{1}$ Michael J. Firbank, ${ }^{1}$ Hrishikesh Kumar, ${ }^{2}$ Payel Chatterjee, ${ }^{2}$ \\ Titas Chakraborty, ${ }^{2}$ Alakananda Dutt ${ }^{2}$ and John-Paul Taylor ${ }^{1}$ \\ ${ }^{1}$ Institute of Neuroscience, Newcastle University, Campus for Ageing and Vitality, Newcastle University, Newcastle upon Tyne NE4 5PL, UK \\ ${ }^{2}$ Institute of Neurosciences Kolkata, AfC Bose Road, Kolkata 700017, West Bengal, India
}

Background: Individuals with Lewy body dementia (LBD) typically exhibit impairments in attentional and executive function. Current pharmacological treatments have limited efficacy, with associated side effects. Transcranial direct current stimulation (tDCS) may represent an alternative treatment, as cognitive improvements have been demonstrated in healthy individuals. However, no studies to date have assessed the feasibility of tDCS in an LBD population. The aim of this preliminary study, therefore, was to assess the tolerability of tDCS, as well as its effects upon attentional and visuoperceptual performance, in LBD patients.

Methods: Thirteen participants completed attentional (simple reaction time, choice reaction time, and digit vigilance) and forced-choice visuoperceptual (angle and motion perception) tasks before and after one 20-min session of active tDCS $\left(0.08 \mathrm{~mA} / \mathrm{cm}^{2}\right)$. The anodal electrode was applied to the left dorsolateral prefrontal cortex and the cathodal electrode was applied to the right deltoid. Attentional (task accuracy and reaction time to correct answers) and visuoperceptual (task accuracy and difficulty) outcome measures were compared using paired t-tests.

Results: All participants tolerated stimulation and did not report any side effects during or immediately after stimulation. Post-stimulation improvements were observed in the choice reaction time (increased percentage of correct answers; $\mathrm{p}=0.01$ ) and digit vigilance (reduced mean reaction time to correct answers; $\mathrm{p}=0.02$ ) attention tasks. Visuoperceptual task performance did not improve (all p-values $>0.05$ ).

Conclusions: Attentional, but not visuoperceptual, improvements were observed following stimulation in LBD patients. Larger-scale, placebo-controlled trials are needed to confirm whether tDCS is a useful treatment option for attentional deficits in LBD.

Key words: Lewy body dementia, dementia with Lewy bodies, Parkinson's disease with dementia, tDCS, transcranial direct current stimulation, attention

\section{Introduction}

Lewy body dementias (LBDs) include dementia with Lewy bodies (DLB) and Parkinson's disease with dementia (PDD). Individuals with DLB and PDD share a common underlying alpha-synuclein neuropathology and display similar neuropsychological impairments, including attentional and visuoperceptual deficits (McKeith et al., 2005; Emre et al., 2007; Lippa et al., 2007).

Correspondence should be addressed to: Dr. Greg J. Elder, Institute of Neuroscience, Campus for Ageing and Vitality, Newcastle University, Newcastle upon Tyne NE4 5PL, UK. Phone: $+44(0) 191208$ 1125. Email: greg.elder@ncl.ac.uk. Received 19 Jan 2015; revision requested 7 Jun 2015; revised version received 12 Jun 2015; accepted 27 Jun 2015. First published online 7 August 2015.
The treatment of attentional and cognitive impairments in LBD is typically limited to the use of cholinesterase inhibitors (McKeith et al., 2000; Emre et al., 2004); however, due to the limited efficacy of these agents and their associated side effects, alternative treatments are urgently needed. One potentially promising nonpharmacological intervention is transcranial direct current stimulation (tDCS), a simple, non-invasive, and inexpensive technique in which a weak electrical current is delivered to the brain through two electrodes soaked in saline or conductive gel. This approach has a modulatory effect upon cortical excitability, where these effects are polaritydependent: anodal stimulation typically increases 
and cathodal stimulation typically decreases the underlying neural membrane potential (Stagg and Nitsche, 2011).

The application of tDCS to the dorsolateral prefrontal cortex (DLPFC) has been shown to enhance cognitive functions including working memory, visuomotor co-ordination and decisionmaking in healthy individuals (Antal et al., 2004; Fregni et al., 2005; Fecteau et al., 2007; Zaehle et al., 2011). Importantly, beneficial cognitive effects following DLPFC stimulation have also been demonstrated in relevant clinical populations, including patients with Alzheimer's disease and Parkinson's disease (Elder and Taylor, 2014). Additionally, these positive effects may persist beyond the immediate period of stimulation. For example, Doruk and colleagues (2014) recently demonstrated that in patients with Parkinson's disease, ten consecutive sessions of anodal tDCS delivered to the left or right DLPFC led to short-term and longer-term (measured one month post-stimulation) improvements in executive functioning, compared with placebo stimulation.

As attentional and executive function deficits are observed within the LBDs, this is suggestive of frontal dysfunction (Johns et al., 2009). This is supported by neuropsychological studies which have observed impairments in frontally dependent attentional and executive tasks in LBD (Ballard et al., 2001; Calderon et al., 2001; Aarsland et al., 2003); pathologically, Lewy bodies are commonly found in the anterior frontal and temporal regions (Yoshimura, 1983; Kuzuhara and Yoshimura, 1993), and in addition, neuroimaging studies have shown that frontal atrophy is a feature of LBD (Burton et al., 2002, 2004). Thus, there is a broad context to suggest that there may be a reduced top-down influence of the frontal cortex in LBD. Previous studies have observed beneficial effects of DLPFC stimulation upon attention and executive functioning (Fecteau et al., 2007; Gladwin et al., 2012), and the left DLPFC has been implicated in top-down cognitive control (MacDonald et al., 2000). Given this empirical and theoretical basis, we therefore hypothesized that the application of anodal tDCS to the DLPFC might benefit attentional and executive function in individuals with LBD. However, to date, the feasibility and tolerability, or the potential benefits of tDCS upon attentional and executive function, have not been examined in an LBD patient group.

Therefore, the primary aim of this preliminary study was to assess the feasibility of using tDCS in individuals with LBD, and to investigate the effects of tDCS upon attentional function in LBD.

\section{Methods}

\section{Participants}

Sixteen participants who met diagnostic criteria for either probable DLB or PDD (McKeith et al., 2005; Emre et al., 2007), as assessed by two experienced clinicians (J.P.T. and H.K.), were recruited from the movement disorders service at the Institute of Neurosciences, Kolkata, India. The diagnosis was verified by detailed physical, neurological, and neuropsychiatric examinations, including hematology and biochemistry screening, thyroid function, syphilis serology, vitamin B12 and folate level tests, and chest X-rays. Structural imaging (magnetic resonance imaging or computed tomography scans) were performed on six of the 16 participants, when clinically suggested, in order to exclude other neurodegenerative etiologies.

Participants were included if they were $\geq 60$ years of age; had a Mini-Mental State Examination (MMSE; Folstein et al., 1975) score of $\geq 16$; and were stable on anti-Parkinsonian medication or cholinesterase inhibitors for a period of one month prior to participation. Exclusion criteria included skin allergies; a history of excess alcohol intake; concurrent major psychiatric illness; significant/severe physical illness or comorbidities; other neurological disorders; current/previous visual impairment due to glaucoma, cataract, or macular degeneration; metallic or electronic implants; and significant motor fluctuations. All participants provided written informed consent and the study was approved by the local ethics committee.

\section{Measures}

Participants completed several clinical measures of cognitive and motor function prior to stimulation in order to provide information regarding disease severity. These included the MMSE (Folstein et al., 1975), the Montreal Cognitive Assessment (MoCA; Nasreddine et al., 2005) and Part III of the Unified Parkinson's Disease Rating Scale (UPDRS-III; Goetz et al., 2008).

\section{Medication use}

Information regarding the use of cholinesterase inhibitors, anti-depressant and anti-Parkinsonian medication was also obtained. The antiParkinsonian medication equivalent levodopa dose was calculated, using levodopa conversion formulae reported elsewhere (Tomlinson et al., 2010), and is expressed in milligrams (mg).

\section{Procedure}

Participants completed one brief practice session of the attentional and visuoperceptual tasks, consisting 


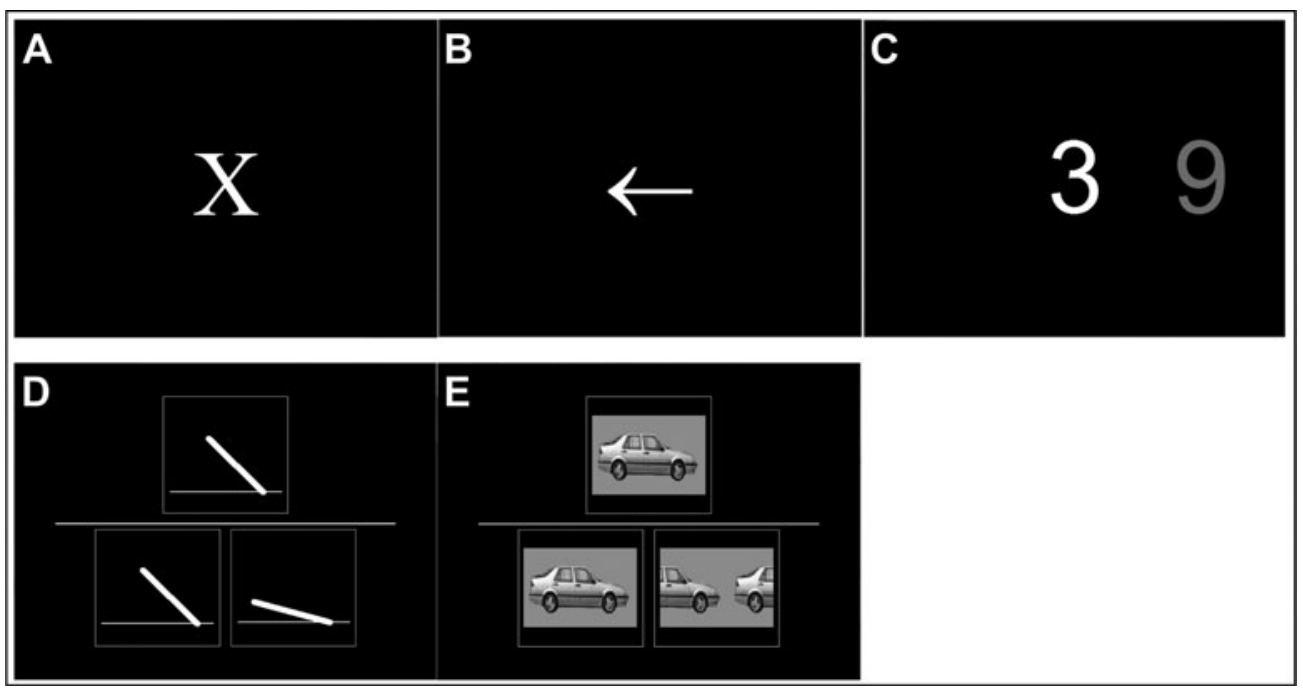

Figure 1. Attentional and visuoperceptual tasks. Task A (simple reaction time): a target $(\mathrm{X})$ was displayed (maximum 3,000 ms with a varying inter-stimulus interval) and participants were required to respond as quickly as possible. Task $B$ (choice reaction time): Participants were required to respond to an arrow, which pointed left or right (displayed for a maximum of 3,000 ms) as quickly as possible and indicating the direction in which the arrow pointed. Task C (digit vigilance): Participants were required to respond to the target digit (9; displayed on the right of the screen) when the digit displayed in the center of the screen (0-9; cycling at a rate of $500 \mathrm{ms)} \mathrm{matched} \mathrm{the}$ target. Tasks D (angle) and E (motion perception): Participants were required to indicate whether the item displayed on the bottom left or the bottom right matched the target item (displayed in the top center of the screen) in terms of the angle (Task D) or the speed of motion (Task E). For both visuoperceptual tasks (Tasks D and E), the difficulty levels for both tasks were adjusted depending on whether the previous trial was correct, where a correct answer increased the difficulty by one step and an incorrect answer reduced the difficulty by three steps.

of five practice trials per task. Participants then completed one full run of the computer tasks (lasting for approximately $10 \mathrm{~min}$ ) immediately prior to stimulation, and repeated the tasks 10 min post-stimulation. Participants were tested in their "on" state with regard to their Parkinsonism; this was verified by ensuring that dopaminergic medication had been administered in the previous 2 $\mathrm{h}$ and by assessment of motor function immediately prior to stimulation.

Three attentional (simple reaction time (SRT); choice reaction time (CRT); digit vigilance (DV)); and two forced-choice visuoperceptual (angle and motion perception) tasks were used; these tasks have previously been demonstrated to be differentially affected in LBD patients compared with controls and other dementia groups (Wesnes et al., 2002; Mosimann et al., 2004). These visuoperceptual tasks are not dependent upon prefrontal regions (Wood et al., 2011) and performance was therefore hypothesized to be unlikely to be affected by DLPFC stimulation. Full details of the tasks are described elsewhere (Mosimann et al., 2004; Wood et al., 2011; 2013) and in Figure 1. Tasks were presented on a laptop PC and responses were recorded using response button boxes, which were held either in the dominant hand or in both hands, depending on the task.

\section{Transcranial direct current stimulation}

A single session of stimulation $(2.8 \mathrm{~mA})$ was delivered for 20 min using an Eldith DC Stimulator (Magstim, Whitland, UK), through two $35-\mathrm{cm}^{2}$ electrodes in $0.9 \%$ saline-soaked bags (equivalent current density: $0.08 \mathrm{~mA} / \mathrm{cm}^{2}$ ). This level of current density has been previously used, with no reported adverse effects, in other dementia cohort studies (Huey et al., 2007; Cotelli et al., 2014). The anodal electrode was placed over the left DLPFC (50\% between $\mathrm{F}_{3}$ and $\mathrm{FP}_{1}$ ) using the International 10-20 system (Jasper, 1958). The cathodal electrode was placed on the right deltoid muscle. All participants received active stimulation. After the stimulation session, participants were asked to provide feedback regarding their experience of the stimulation; similarly, patients and their families were contacted $24 \mathrm{~h}$ post-stimulation and were asked whether any residual side effects were present.

\section{Statistical analysis}

Attentional and visuoperceptual task performances were compared before and after stimulation using paired t-tests. Attentional (SRT, CRT, and DV) task outcome measures included the percentage of correct answers and the mean reaction time (RT) to correct answers. Visuoperceptual outcome 
Table 1. Participant demographic and assessment data $(n=13)$

\begin{tabular}{llc}
\hline & DLB $(n=5)$ & PDD $(n=8)$ \\
Age (years): mean (SD) & $65.00(7.71)$ & $64.63(8.16)$ \\
Gender: (male/female) & $3 / 2$ & $7 / 1$ \\
Duration of motor symptoms (years since onset): mean (SD) & $3.80(2.39)$ & $11.75(3.73)$ \\
Duration of cognitive symptoms (years since onset): mean (SD) & $4.00(2.91)$ & $2.63(1.51)$ \\
Presence of visual hallucinations: $n$ (\%) & $2(40)$ & $7(87.5)$ \\
Presence of delusions: $n$ (\%) & $3(60)$ & $7(87.5)$ \\
Presence of apathy: $n$ (\%) & $4(80)$ & $8(100)$ \\
Presence of excessive daytime sleepiness: $n(\%)$ & $2(40)$ & $4(50)$ \\
Presence of depression or anxiety: $n(\%)$ & $3(60)$ & $6(75)$ \\
Presence of cognitive fluctuations: $n(\%)$ & $3(60)$ & $6(75)$ \\
MMSE: mean (SD) & $20.60(3.05)$ & $22.00(2.56)$ \\
MoCA: mean (SD) & $17.60(3.65)$ & $19.38(2.45)$ \\
UPDRS-III: mean (SD) & $12.75(5.56)$ & $28.50(9.44)$ \\
On cholinesterase inhibitors: $n(\%)$ & $1(20)$ & $0(0)$ \\
On anti-Parkinsonian medication, $n(\%)$ & $5(100)$ & $8(100)$ \\
On anti-depressant medication, $n(\%)$ & $0(0)$ & $3(37.5)$ \\
Levodopa equivalent dose (mg): mean $(S D)$ & $320.00(168.08)$ & $935.50(599.38)$ \\
\hline
\end{tabular}

Notes: DLB: Dementia with Lewy bodies; PDD: Parkinson's disease with dementia; MMSE: Mini-Mental State Examination; MoCA: Montreal Cognitive Assessment; UPDRS-III: Unified Parkinson's Disease Rating Scale Motor Subscale.

${ }^{a}$ UPDRS-III data missing for one participant.

measures included the percentage of correct answers and the task difficulty, expressed either as degree values (angle task, where a lower degree value indicates better performance) or relative speed values (cars task, where a lower relative speed indicates a better performance). Outliers ( $\geq 2$ SD) were removed from each RT task. As the aim of the current preliminary study was to assess the feasibility and tolerability of tDCS in LBD participants, analyses were not adjusted for multiple comparisons. Effect sizes are reported as Cohen's $d_{z}$.

\section{Results}

Three participants were excluded from the study prior to stimulation, due to a poor understanding of task instructions. This resulted in a final sample of 13 participants (8 PDD; 5 DLB; Mean, $M_{\text {age }}$ $=64.77$ years, $\mathrm{SD}_{\mathrm{age}}=7.66$ years). Demographic details are included in Table 1. All participants tolerated stimulation and did not report any side effects, other than a brief tingling sensation underneath the electrodes, during or immediately after stimulation. No adverse events were reported.

The percentage of CRT correct answers significantly increased following stimulation $(\mathrm{t}(12)=$ $\left.-2.98, \mathrm{p}=0.01, d_{z}=0.83\right)$. DV improvements were also shown, where the mean RT to correct answers $\left(\mathrm{t}(11)=2.77, \mathrm{p}=0.02, d_{z}=0.80\right)$ was significantly reduced following stimulation. Eleven participants $(84.6 \%)$ showed a positive CRT task response, displaying an increased percentage of correct answers following stimulation, and nine participants $(69.2 \%)$ showed a positive DV task response, with a reduced mean RT to correct answers following stimulation. No other significant post-stimulation differences were shown in the remaining attentional or visuoperceptual tasks (all p-values $>0.05)$. The results are summarized in Table 2.

\section{Discussion}

This preliminary study indicates that the use of tDCS, with a current density of 0.08 $\mathrm{mA} / \mathrm{cm}^{2}$, is both feasible and well-tolerated in a group of individuals with $\mathrm{LBD}$, as no residual post-stimulation side effects were reported by participants or their families. This study also demonstrated that a single 20 -min session of tDCS, applied to the left DLPFC, led to post-stimulation improvements to some aspects of attentional, but not visuoperceptual, function in LBD patients. In particular, there were improvements in CRT accuracy and in DV reaction times, although there was also a broader tendency for post-stimulation improvements in all other CRT and DV attentional measures. This differential benefit is likely to be due to the placement of electrode over the DLPFC rather than parietal or visual areas, since previous studies have shown that stimulation delivered to the DLPFC enhances working memory and decision-making (Fregni et al., 2005; Fecteau et al., 2007), and in the case of left DLPFC stimulation, can enhance attentional function (Gladwin et al., 
Table 2. Attention and visuoperceptual task performance pre- and post-tDCS stimulation $(n=13)$

\begin{tabular}{|c|c|c|c|c|c|}
\hline \multirow[b]{2}{*}{ ATTENTION TASKS } & \multicolumn{2}{|c|}{ PRE-STIMULATION } & \multicolumn{3}{|c|}{ POST-STIMULATION } \\
\hline & MEAN & SD & MEAN & SD & $\operatorname{EFFECT~SIZE~}\left(d_{z}\right)$ \\
\hline SRT: correct answers (\%) & 94.87 & 7.77 & 94.36 & 8.86 & 0.13 \\
\hline SRT: mean RT (ms; correct answers) ${ }^{\mathrm{a}}$ & 454.65 & 130.63 & 453.65 & 139.41 & 0.01 \\
\hline CRT: correct answers (\%) & $71.79^{*}$ & 28.34 & $87.69^{*}$ & 20.92 & 0.83 \\
\hline CRT: mean RT (ms; correct answers) ${ }^{\mathrm{a}}$ & 620.44 & 202.90 & 608.78 & 157.18 & 0.12 \\
\hline DV: correct answers $(\%)$ & 75.64 & 15.60 & 81.41 & 26.53 & 0.30 \\
\hline DV: mean RT (ms; correct answers) ${ }^{\mathrm{a}}$ & $632.85^{*}$ & 100.16 & $582.93^{*}$ & 58.42 & 0.80 \\
\hline \multicolumn{6}{|l|}{ Visuoperceptual tasks } \\
\hline Angle: correct answers (\%) & 85.11 & 7.36 & 84.88 & 7.15 & 0.02 \\
\hline Angle: difficulty (degrees) ${ }^{\mathrm{b}}$ & 24.01 & 16.56 & 23.63 & 18.99 & 0.02 \\
\hline Motion: correct answers (\%) & 70.36 & 13.18 & 75.90 & 11.49 & 0.44 \\
\hline Motion: difficulty (relative speed) ${ }^{c}$ & 2.61 & 0.97 & 2.56 & 1.00 & 0.07 \\
\hline
\end{tabular}

Notes: SRT: simple reaction time; CRT: choice reaction time; DVT: digit vigilance; ms: milliseconds; RT: reaction time.

${ }^{\mathrm{a}} n=12$.

${ }^{\mathrm{b}}$ Lower degree value indicates better performance.

${ }^{\mathrm{c}}$ Lower relative speed indicates better performance.

${ }^{*} \mathrm{p}<0.05$.

2012). Speculatively, our data suggest that as the left DLPFC has a suggested role in topdown cognitive control (MacDonald et al., 2000), anodal stimulation, which can modulate cortical excitability by increasing the membrane potential (Stagg and Nitsche, 2011), might help improve the degree of top-down influence of the left DLPFC in LBD.

Whilst not placebo-controlled, the results of the present study may also support the notion that visuoperceptual performance in LBD is less dependent on fronto-executive function; posterior cortical function may be more relevant for these tasks and this would be consistent with the parieto-occipital deficits apparent in LBD (Colloby et al., 2002). It is likely that task-specific electrode montages will be needed to improve visuoperceptual function in LBD; the application of anodal stimulation to right parietal areas has been shown to result in visuoperceptual improvements within healthy individuals (Bolognini et al., 2010). Therefore, the efficacy of anodal stimulation to parietal areas should be tested within LBD.

Limitations of the current study include the absence of a sham condition and the lack of correction for multiple comparisons in our analyses. However, by its nature, this study is exploratory in terms of highlighting potential benefits, determining tolerability, and helping to inform stimulation parameters; the details of which are currently lacking in LBD and can be determined by studies with small sample sizes and studies that are open-label in design (Elder and Taylor, 2014). Although the present study assessed a small sample of patients, a current density of $0.08 \mathrm{~mA} / \mathrm{cm}^{2}$, delivered for a period of $20 \mathrm{~min}$, was shown to be both acceptable and tolerable in LBD patients. Whilst this level of current density has previously been used in other dementia cohort studies (Huey et al., 2007; Cotelli et al., 2014), this has not been the case in LBD, and importantly, these stimulation parameters could be used in future placebo-controlled studies in LBD.

While post-stimulation performance improvements were not observed across all task measures, the influence of training effects cannot be excluded from the present study. For this reason, placebocontrolled trials are necessary to confirm these results. However, the present study contributes important pilot data in LBD, as it has been recommended that the results of all studies, including those which are negative, are reported in order to advance the clinical utility of tDCS and to allow the inclusion of eligible studies in future meta-analyses (Elder and Taylor, 2014).

Finally, it was not possible to examine whether stimulation differentially benefits DLB or PDD patients in the present study; certainly whilst there is significant overlap neuropathologically and clinically between both conditions (McKeith et al., 2005; Emre et al., 2007; Lippa et al., 2007), differences between the two groups have been suggested (Park et al., 2011). Nevertheless, these preliminary data suggest that tDCS may benefit both patient populations, thus supporting largescale trials in both patient groups.

In conclusion, this study indicates that tDCS is feasible and is well tolerated in an LBD population, adding to the current lack of data in this patient group. This study also provides tentative evidence 
to suggest that the delivery of tDCS to the left DLPFC might benefit attentional function in LBD. However, larger placebo-controlled trials with repeated stimulation sessions are needed, and are now justified, in an LBD population to confirm this; promising data in PD have suggested that tDCS can offer sustained benefits upon executive functioning (Doruk et al., 2014) and it would be relevant to explore if these effects are carried over into LBD.

\section{Conflict of interest}

None.

\section{Description of authors' roles}

G. J. Elder conceived and designed the study, analyzed and interpreted the data, and drafted the manuscript. M. J. Firbank analyzed and interpreted the data, and revised the manuscript. H. Kumar, P. Chatterjee, T. Chakraborty, and A. Dutt conceived and designed the study, assisted with the recruitment of participants, and revised the manuscript. J-P Taylor conceived and designed the study, analyzed and interpreted the data and revised the manuscript. All authors have approved the final version of the manuscript.

\section{Acknowledgments}

The research was supported by an Intermediate Clinical Fellowship to Dr. J-P Taylor (WT088441 $\mathrm{MA}$ ) and the National Institute of Health Research (NIHR) Newcastle Biomedical Research Unit based at Newcastle upon Tyne Hospitals NHS Foundation Trust and Newcastle University. The views expressed are those of the authors and not necessarily those of the NHS, the NIHR, or the Department of Health. The funders had no role in the study design, in the collection, analysis, and interpretation of data, in the writing of the manuscript, or in the decision to submit the manuscript for publication.

\section{References}

Aarsland, D., Litvan, I., Salmon, D., Galasko, D., Wentzel-Larsen, T. and Larsen, J. P. (2003). Performance on the dementia rating scale in Parkinson's disease with dementia and dementia with Lewy bodies: comparison with progressive supranuclear palsy and Alzheimer's disease. Fournal of Neurology, Neurosurgery and Psychiatry, 74, 1215-1220.

Antal, A., Nitsche, M. A., Kruse, W., Kincses, T. Z., Hoffmann, K.-P. and Paulus, W. (2004). Direct current stimulation over V5 enhances visuomotor coordination by improving motion perception in humans. Fournal of Cognitive Neuroscience, 16, 521-527.

Ballard, C. et al. (2001). Attention and fluctuating attention in patients with dementia with Lewy bodies and Alzheimer disease. Archives of Neurology, 58, 977-982.

Bolognini, N., Fregni, F., Casati, C., Olgiati, E. and Vallar, G. (2010). Brain polarization of parietal cortex augments training-induced improvement of visual exploratory and attentional skills. Brain Research, 1349, 76-89.

Burton, E. J., McKeith, I. G., Burn, D. J., Williams, E. D. and O'Brien, J. T. (2004). Cerebral atrophy in Parkinson's disease with and without dementia: a comparison with Alzheimer's disease, dementia with Lewy bodies and controls. Brain, 127, 791-800.

Burton, E. J. et al. (2002). Patterns of cerebral atrophy in dementia with Lewy bodies using voxel-based morphometry. Neuroimage, 17, 618-630.

Calderon, J., Perry, R. J., Erzinclioglu, S. W., Berrios, G. E., Dening, T. R. and Hodges, J. R. (2001). Perception, attention, and working memory are disproportionately impaired in dementia with Lewy bodies compared with Alzheimer's disease. Fournal of Neurology, Neurosurgery and Psychiatry, 70, 157-164.

Colloby, S. J. et al. (2002). A comparison of (99 $\mathrm{m})$ Tc-HMPAO SPET changes in dementia with Lewy bodies and Alzheimer's disease using statistical parametric mapping. European fournal of Nuclear Medicine and Molecular Imaging, 29, 615-622.

Cotelli, M. et al. (2014). Anodal tDCS during face-name associations memory training in Alzheimer's patients. Frontiers in Aging Neuroscience, 6, 38.

Doruk, D., Gray, Z., Bravo, G. L., Pascual-Leone, A. and Fregni, F. (2014). Effects of tDCS on executive function in Parkinson's disease. Neuroscience Letters, 582, 27-31.

Elder, G. J. and Taylor, J. P. (2014). Transcranial magnetic stimulation and transcranial direct current stimulation: treatments for cognitive and neuropsychiatric symptoms in the neurodegenerative dementias? Alzheimer's Research and Therapy, 6, 74 .

Emre, M. et al. (2004). Rivastigmine for dementia associated with Parkinson's disease. New England fournal of Medicine, 351, 2509-2518.

Emre, M. et al. (2007). Clinical diagnostic criteria for dementia associated with Parkinson's disease. Movement Disorders, 22, 1689-1707.

Fecteau, S., Knoch, D., Fregni, F., Sultani, N., Boggio, P. and Pascual-Leone, A. (2007). Diminishing risk-taking behavior by modulating activity in the prefrontal cortex: a direct current stimulation study. Fournal of Neuroscience, 27, 12500-12505.

Folstein, M. F., Folstein, S. E. and McHugh, P. R. (1975). "Mini-Mental State": a practical method for grading the cognitive state of patients for the clinician. Fournal of Psychiatric Research, 12, 189-198.

Fregni, F. et al. (2005). Anodal transcranial direct current stimulation of prefrontal cortex enhances working memory. Experimental Brain Research, 166, 23-30.

Gladwin, T. E., den Uyl, T. E., Fregni, F. F. and Wiers, R. W. (2012). Enhancement of selective attention by 
tDCS: interaction with interference in a Sternberg task. Neuroscience Letters, 512, 33-37.

Goetz, C. G. et al. (2008). Movement Disorder Society-sponsored revision of the Unified Parkinson's Disease Rating Scale (MDS-UPDRS): scale presentation and clinimetric testing results. Movement Disorders, 23, 2129-2170.

Huey, E. D. et al. (2007). No effect of DC brain polarization on verbal fluency in patients with advanced frontotemporal dementia. Clinical Neurophysiology, 118, 14171418.

Jasper, H. H. (1958). The ten-twenty electrode system of the international federation. Electroencephalography and Clinical Neurophysiology, 10, 371-375.

Johns, E. K. et al. (2009). Executive functions in frontotemporal dementia and Lewy body dementia. Neuropsychology, 23, 765-777.

Kuzuhara, S. and Yoshimura, M. (1993). Clinical and neuropathological aspects of diffuse Lewy body disease in the elderly. Advances in Neurology, 60, 464-469.

Lippa, C. F. et al. (2007). DLB and PDD boundary issues: diagnosis, treatment, molecular pathology, and biomarkers. Neurology, 68, 812-819.

MacDonald, A. W., Cohen, J. D., Stenger, V. A. and Carter, C. S. (2000). Dissociating the role of the dorsolateral prefrontal and anterior cingulate cortex in cognitive control. Science, 288, 1835-1838.

McKeith, I. et al. (2000). Efficacy of rivastigmine in dementia with Lewy bodies: a randomised, double-blind, placebo-controlled international study. Lancet, 356, 2031-2036.

McKeith, I. G. et al. (2005). Diagnosis and management of dementia with Lewy bodies: third report of the DLB consortium. Neurology, 65, 1863-1872.

Mosimann, U. P., Mather, G., Wesnes, K. A., O'Brien, J. T., Burn, D. J. and McKeith, I. G. (2004). Visual perception in Parkinson's disease dementia and dementia with Lewy bodies. Neurology, 63, 2091-2096.
Nasreddine, Z. S. et al. (2005). The Montreal Cognitive Assessment, MoCA: a brief screening tool for mild cognitive impairment. Fournal of the American Geriatrics Society, 53, 695-699.

Park, K. W., Kim, H. S., Cheon, S. M., Cha, J. K., Kim, S. H. and Kim, J. W. (2011). Dementia with Lewy bodies versus Alzheimer's disease and Parkinson's disease dementia: a comparison of cognitive profiles. Fournal of Clinical Neurology, 7, 19-24.

Stagg, C. J. and Nitsche, M. A. (2011). Physiological basis of transcranial direct current stimulation. Neuroscientist, 17, 37-53.

Tomlinson, C. L., Stowe, R., Patel, S., Rick, C., Gray, R. and Clarke, C. E. (2010). Systematic review of levodopa dose equivalency reporting in Parkinson's disease. Movement Disorders, 25, 2649-2653.

Wesnes, K. A. et al. (2002). Effects of rivastigmine on cognitive function in dementia with lewy bodies: a randomised placebo-controlled international study using the cognitive drug research computerised assessment system. Dementia and Geriatric Cognitive Disorders, 13, 183-192.

Wood, J. S., Firbank, M. J., Mosimann, U. P., Taylor, J.-P. and O'Brien, J. T. (2011). Development of a novel fMRI compatible visual perception prototype battery to test older people with and without dementia. Fournal of Geriatric Psychiatry and Neurology, 24, 73-83.

Wood, J. S. et al. (2013). Testing visual perception in dementia with Lewy bodies and Alzheimer's disease. American fournal of Geriatric Psychiatry, 21, 501-508.

Yoshimura, M. (1983). Cortical changes in the Parkinsonian brain: a contribution to the delineation of "diffuse Lewy body disease." Fournal of Neurology, 229, 17-32.

Zaehle, T., Sandmann, P., Thorne, J., Jancke, L. and Herrmann, C. (2011). Transcranial direct current stimulation of the prefrontal cortex modulates working memory performance: combined behavioral and electrophysiological evidence. BMC Neuroscience, 12, 2. 